\title{
Robustness of a compact endfire personal audio system against scattering effects $(L)$
}

\author{
Zhen Tu and Jing Lu ${ }^{\text {a) }}$ \\ Key Lab of Modern Acoustics, Institute of Acoustics, Nanjing University, Nanjing 210093, China \\ Xiaojun Qiu \\ Faculty of Engineering and Information Technology, University of Technology, Sydney, \\ New South Wales 2007, Australia
}

(Received 17 July 2016; revised 26 September 2016; accepted 30 September 2016; published online 17 October 2016)

\begin{abstract}
Compact loudspeaker arrays have wide potential applications as portable personal audio systems that can project sound energy to specified regions. It is meaningful to investigate the scattering effects on the array performance since the scattering of the users' heads is inevitable in practice. A five-channel compact endfire array is established and the regularized acoustic contrast control method is evaluated for the scenarios of one moving listener and one listener fixed in the bright zone while another listener moves along the evaluation region. Both simulations and experiments verify that the scattering has limited influence on the directivity of the endfire array.
\end{abstract}

(C) 2016 Acoustical Society of America. [http://dx.doi.org/10.1121/1.4964752]

Pages: $2720-2724$

\section{INTRODUCTION}

Personal audio systems, which use loudspeaker arrays to obtain proper sound pressure around listeners in one or several regions while minimizing the interference to listeners in other regions, have attracted many researchers' interest. ${ }^{1,2}$ The acoustic contrast control (ACC) method $^{3}$ and the pressure matching method (PMM) $)^{4}$ are two most commonly used sound field control strategies. In principle, the ACC method yields largest acoustic "contrast" between the bright zone and the dark zone, while the PMM leads to better reproduction performance in the desired field. Many array structures have been investigated and the balance between different control strategies has been discussed. ${ }^{5-8}$

Among different topologic array structures, the compact linear personal audio system is of particular interest due to its potential applications in portable communication devices. The algorithm for the compact linear array with bright zone defined in the endfire direction was analyzed by Elliott et al., ${ }^{8}$ and the experimental evaluation of a two-source array mounted on a mobile phone-sized device was given later. ${ }^{9}$ It was pointed out that the directivity of the compact linear array can be further improved by using phase shift loudspeakers. $^{10}$

In practical applications, the robustness of the control algorithms is important because the loudspeaker positions bias, the mismatch between loudspeakers and the variation of acoustic transfer functions all deteriorate the performance of the system. ${ }^{11,12}$ It has been investigated that when used in a practical classroom or an audio room, the performance of the array system will be considerably influenced by the room reverberation effects. ${ }^{13}$ For compact arrays that are utilized in a short distance, the head scattering effects are usually inevitable since the user is often close to the array system. It

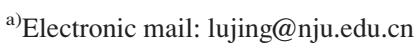

has been found that the scattering of one head deteriorates the array performance of a linear array with the square bright zone set at the broadside direction and two rectangle dark zones at the sides. ${ }^{14}$ However, the influence of the head scattering on the performance of compact arrays with bright zone at the endfire direction has not been discussed. Furthermore, for the common application scenario where more than one listener is around the array, it is important to evaluate the difference between the sound pressures at the listeners' ears.

In this paper, based on a five-channel compact linear loudspeaker array model, the regularized ACC method is employed to project sound energy to the endfire direction of the loudspeaker array and to optimize the control zones for better broadband performance. Both one-head and two-head scattering models are used in simulations and experiments to investigate the robustness of the compact endfire system against the scattering effects.

\section{THE COMPACT ENDFIRE ARRAY MODEL}

\section{A. Description of the endfire array model}

The five-channel compact endfire array system in free field is shown in Fig. 1(a), where the space between two adjacent loudspeakers is $\Delta x=0.045 \mathrm{~m}$. The origin of the coordinates is at the center loudspeaker, and the radius of the evaluation zone is $r=1.0 \mathrm{~m}$ with 72 sampling points evenly distributed on the circle. The frequency band $500-3500 \mathrm{~Hz}$ that covers most energy of speech signals is of primary concern.

The total sound pressure at position $\mathbf{r}$ can be expressed as ${ }^{6}$

$$
p(\mathbf{r})=\sum_{n=1}^{N} H\left(\mathbf{r} \mid \mathbf{r}_{L n}\right) q_{n},
$$


where $q_{n}$ is the source strength of the $n$th loudspeaker, $N=5$ is the number of the loudspeakers, and $H\left(\mathbf{r} \mid \mathbf{r}_{L n}\right)$ is the transfer function between the $n$th loudspeaker at $\mathbf{r}_{L n}$ and the sampling point at $\mathbf{r}$. In free field, the transfer function can be expressed as ${ }^{6}$

$$
H\left(\mathbf{r} \mid \mathbf{r}_{L n}\right)=\frac{e^{-i k\left|\mathbf{r}-\mathbf{r}_{L n}\right|}}{\left|\mathbf{r}-\mathbf{r}_{L n}\right|}
$$

There are $M$ discrete sampling points centered at the endfire direction in the bright zone, denoted as $\mathbf{r}_{\mathrm{b} 1}, \mathbf{r}_{\mathrm{b} 2}, \ldots$, $\mathbf{r}_{\mathrm{b} M}$. The remaining $K$ points are in the dark zone, denoted as $\mathbf{r}_{\mathrm{d} 1}, \mathbf{r}_{\mathrm{d} 2}, \ldots, \mathbf{r}_{\mathrm{d} K}$. The corresponding transfer functions can be expressed in matrix form as

$$
\begin{gathered}
\mathbf{H}_{\mathrm{b}}=\left[\begin{array}{ccc}
H\left(\mathbf{r}_{\mathrm{b} 1} \mid \mathbf{r}_{L 1}\right) & \cdots & H\left(\mathbf{r}_{\mathrm{b} 1} \mid \mathbf{r}_{L N}\right) \\
\vdots & \ddots & \vdots \\
H\left(\mathbf{r}_{\mathrm{b} M} \mid \mathbf{r}_{L 1}\right) & \cdots & H\left(\mathbf{r}_{\mathrm{b} M} \mid \mathbf{r}_{L N}\right)
\end{array}\right], \\
\mathbf{H}_{\mathrm{d}}=\left[\begin{array}{ccc}
H\left(\mathbf{r}_{\mathrm{d} 1} \mid \mathbf{r}_{L 1}\right) & \cdots & H\left(\mathbf{r}_{\mathrm{d} 1} \mid \mathbf{r}_{L N}\right) \\
\vdots & \ddots & \vdots \\
H\left(\mathbf{r}_{\mathrm{d} K} \mid \mathbf{r}_{L 1}\right) & \cdots & H\left(\mathbf{r}_{\mathrm{d} K} \mid \mathbf{r}_{L N}\right)
\end{array}\right],
\end{gathered}
$$

and the source strength can be expressed as

$$
\mathbf{q}=\left[\begin{array}{llll}
q_{1} & q_{2} & \cdots & q_{N}
\end{array}\right]^{\mathrm{T}} .
$$

The sound pressure in different zones can be rewritten in matrix form as

$$
\mathbf{p}_{\mathrm{b}}=\mathbf{H}_{\mathrm{b}} \mathbf{q}, \quad \mathbf{p}_{\mathrm{d}}=\mathbf{H}_{\mathrm{d}} \mathbf{q}
$$

where $\mathbf{p}_{\mathrm{b}}=\left[p\left(\mathbf{r}_{\mathrm{b} 1}\right), \ldots, p\left(\mathbf{r}_{\mathrm{b} M}\right)\right]^{\mathrm{T}}$ and $\mathbf{p}_{\mathrm{d}}=\left[p\left(\mathbf{r}_{\mathrm{d} 1}\right), \ldots, p\left(\mathbf{r}_{\mathrm{d} K}\right)\right]^{\mathrm{T}}$.

To focus sound energy in the bright zone while reducing sound energy in the dark zone, the ACC method is introduced in Ref. 3, where the optimization goal is set as

$$
\max _{\mathbf{q}}\left\{\frac{\mathbf{p}_{\mathrm{b}}^{\mathrm{H}} \mathbf{p}_{\mathrm{b}}}{\mathbf{p}_{\mathrm{d}}^{\mathrm{H}} \mathbf{p}_{\mathrm{d}}}\right\}=\max _{\mathbf{q}}\left\{\frac{\mathbf{q}^{\mathrm{H}} \mathbf{H}_{\mathrm{b}}^{\mathrm{H}} \mathbf{H}_{\mathrm{b}} \mathbf{q}}{\mathbf{q}^{\mathrm{H}} \mathbf{H}_{\mathrm{d}}^{\mathrm{H}} \mathbf{H}_{\mathrm{d}} \mathbf{q}}\right\},
$$
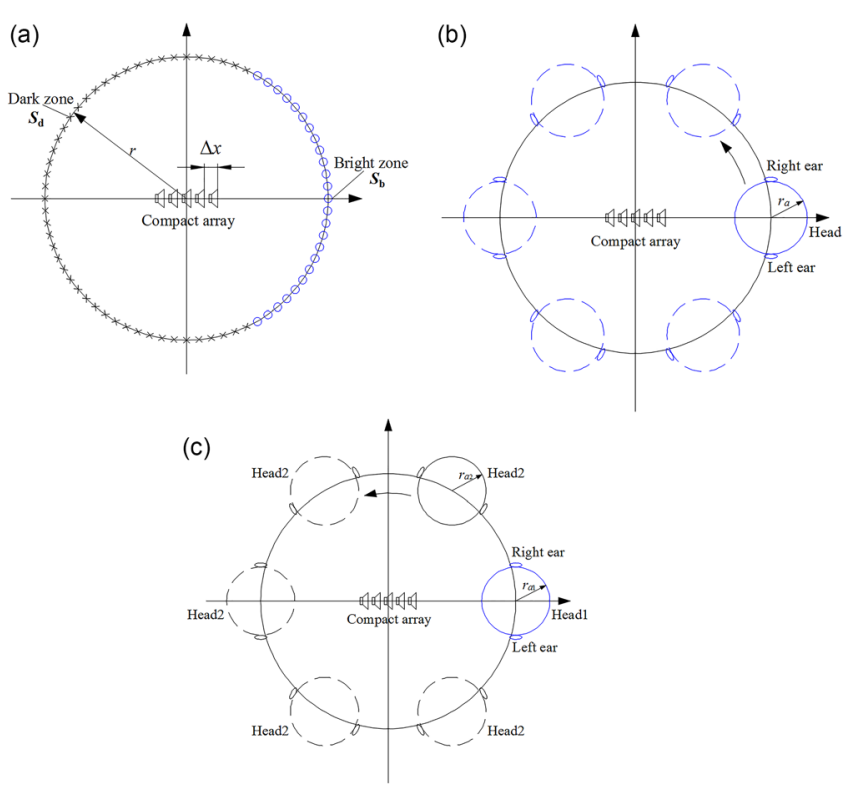

FIG. 1. (Color online) The compact endfire array model with the bright zone and the dark zone. (a) The free field array model, (b) one-head scattering model, and (c) two-head scattering model.

and the solution is found to be proportional to the eigenvector of the matrix $\left(\mathbf{H}_{\mathrm{d}}^{\mathrm{H}} \mathbf{H}_{\mathrm{d}}\right)^{-1}\left(\mathbf{H}_{\mathrm{b}}^{\mathrm{H}} \mathbf{H}_{\mathrm{b}}\right)$, which corresponds to its largest eigenvalue. In practical applications, the matrix $\left(\mathbf{H}_{\mathrm{d}}^{\mathrm{H}} \mathbf{H}_{\mathrm{d}}+\beta \mathbf{I}\right)^{-1}\left(\mathbf{H}_{\mathrm{b}}^{\mathrm{H}} \mathbf{H}_{\mathrm{b}}\right)$ is often used, where $\beta$ is the regularization factor and $\mathbf{I}$ is the identity matrix. The regularization factor is optimized to ensure a low condition number of the matrix $\mathbf{H}_{\mathrm{d}}^{\mathrm{H}} \mathbf{H}_{\mathrm{d}}+\beta \mathbf{I}{ }^{7}{ }^{7}$ so that the robustness of the compact endfire array is improved and the power consumption is limited. ${ }^{8}$

\section{B. Head scattering models}

The user's head is assumed to be a rigid sphere and two typical head scattering scenarios are considered. The first is that the sphere moves along the circle, as depicted in Fig. 1(b), and the second is that one sphere is at the center of the bright zone while the other moves along the circle, as depicted in Fig. 1(c). For the first scenario, the transfer function between the loudspeaker at $\mathbf{r}_{L}=\left(r_{L}, \theta_{L}, \varphi_{L}\right)$ and the sampling point at $\mathbf{r}=(r, \theta, \varphi)$ is ${ }^{15}$

$$
H_{s}\left(\mathbf{r} \mid \mathbf{r}_{L}\right)=\left\{\begin{array}{l}
4 \pi i k \sum_{l=0}^{\infty}\left[j_{l}(k r)-\frac{j_{l}^{\prime}\left(k r_{a}\right)}{h_{l}^{\prime}\left(k r_{a}\right)}\right] h_{l}\left(k r_{L}\right) \sum_{m=-l}^{l} Y_{l}^{m}\left(\theta_{L}, \varphi_{L}\right)^{*} Y_{l}^{m}(\theta, \varphi), \quad r_{\mathrm{a}}<r<r_{L} \\
4 \pi i k \sum_{l=0}^{\infty}\left[j_{l}\left(k r_{L}\right)-\frac{j_{l}^{\prime}\left(k r_{a}\right)}{h_{l}^{\prime}\left(k r_{a}\right)}\right] h_{l}(k r) \sum_{m=-l}^{l} Y_{l}^{m}\left(\theta_{L}, \varphi_{L}\right)^{*} Y_{l}^{m}(\theta, \varphi), \quad r>r_{L}
\end{array}\right.
$$

where $j_{l}(\bullet)$ is the spherical Bessel function, $h_{l}(\bullet)$ is the spherical Hankel function of the first kind, and $Y_{l}^{m}(\bullet)$ is the Laplace spherical harmonics.

For the second scenario, the transfer function between the loudspeaker at $\mathbf{r}_{L}$ and the sampling point at $\mathbf{r}$ can be expressed as ${ }^{16}$

$$
H_{s}\left(\mathbf{r} \mid \mathbf{r}_{L}\right)=H\left(\mathbf{r} \mid \mathbf{r}_{L}\right)+H_{s 1}\left(\mathbf{r} \mid \mathbf{r}_{L}\right)+H_{s 2}\left(\mathbf{r} \mid \mathbf{r}_{L}\right)
$$


where $H\left(\mathbf{r} \mid \mathbf{r}_{L}\right)$ is the free field transfer function, $H_{s 1}\left(\mathbf{r} \mid \mathbf{r}_{L}\right)$ and $H_{s 2}\left(\mathbf{r} \mid \mathbf{r}_{L}\right)$ are the scattering transfer functions of two spheres. The three transfer functions can be expanded using spherical harmonics, and the coefficients can be obtained utilizing the rigid boundary conditions on two spheres.

\section{SIMULATIONS}

The robustness of the system to the head scattering effects is analyzed by comparing results with and without the influence of the head models. For all the scenarios in our work, including the free field scenario, one-head scattering scenario and two-head scattering scenario, the optimal source strength is obtained just using the free field transfer function, which means that the generated signals are the same for all scenarios. However, the transfer function is different to analyze the focusing performance of the compact endfire array under different scenarios. For the free field case shown in Fig. 1(a), the sound pressure at the 72 sampling points are calculated and normalized with respect to the sound pressure at the endfire sampling point. Meanwhile, the commonly used acoustic contrast $(\mathrm{AC})^{3}$

$$
\mathrm{AC}=20 \log _{10}\left(\frac{\mathbf{p}_{\mathrm{b}}^{\mathrm{H}} \mathbf{p}_{\mathrm{b}} / M}{\mathbf{p}_{\mathrm{d}}^{\mathrm{H}} \mathbf{p}_{\mathrm{d}} / K}\right)
$$

is utilized to evaluate the focusing performance of the compact array.

For the one-head scattering case shown in Fig. 1(b), the sound pressure at two ears $p_{\text {left }}$ and $p_{\text {right }}$ with the head center at the 72 sampling points is calculated using Eqs. (5) and (7), then the average sound pressure is obtained by

$$
p=\sqrt{\left(p_{\text {left }}^{2}+p_{\text {right }}^{2}\right) / 2} .
$$

The normalization is carried out using the average sound pressure with head center at the endfire sampling point, and the $\mathrm{AC}$ is calculated using the average sound pressure in the bright and dark zone to investigate the robustness of onehead scattering on the system. The radius of the head is $r_{\mathrm{a}}=0.1 \mathrm{~m}$ throughout this paper.

For the two-head scattering case shown in Fig. 1(c), The center of Head 1 is fixed at the endfire sampling point while the center of Head 2 is placed at 65 permitted sampling points (the center of Head 2 is placed outside of $-15^{\circ}-15^{\circ}$ to ensure non-overlap of Head 1 and Head 2). The sound pressure at two ears of each head is calculated using Eqs. (5) and (8), and the average sound pressure is calculated using Eq. (10). The normalization of the average sound pressure of Head 2 is carried out using the average sound pressure of Head 1. Then the AC is calculated using the average sound pressure in the bright and dark zones. The radius of two heads are $r_{\mathrm{a} 1}=r_{\mathrm{a} 2}=0.1 \mathrm{~m}$ throughout this paper.

The free field directivities of the compact array at two typical frequencies with varied number of bright points $M$ are shown in Figs. 2(a) and 2(b), where it is clear that the performance of the compact array at high frequency changes significantly. To obtain a uniform beamwidth in the bright zone and minimize the sidelobes in the dark

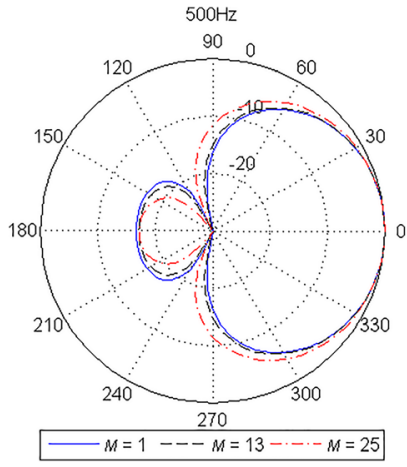

(a)

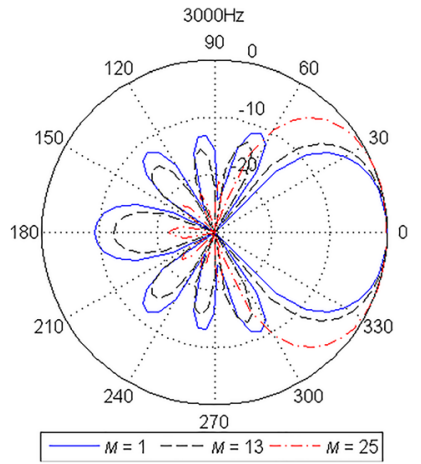

(b)
FIG. 2. (Color online) Single-frequency directivity variation with different number of bright points, (a) $500 \mathrm{~Hz}$, (b) $3000 \mathrm{~Hz}$. The performance with 1 bright point is shown by the solid line, the performance with 13 bright points is shown by the dash line, and the performance with 25 bright points is shown by the dash-dot line.

zone, $M=25$ is used in the following simulations and experiments.

For the realization of the broadband control, a 160-tap finite impulse response (FIR) filter with sampling frequency of $8 \mathrm{kHz}$ is designed for each loudspeaker as follows. ${ }^{17}$ The optimization is carried out in frequency domain using Eq. (6), with $50 \mathrm{~Hz}$ frequency interval. Then the amplitude of the source strength at different frequencies is adjusted to ensure a flat amplitude response in the center of the bright zone, and the adjusted source strength can be expressed as

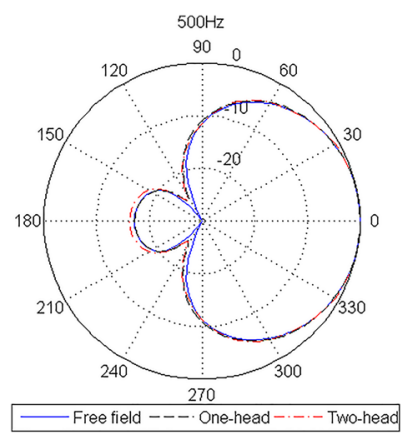

(a)

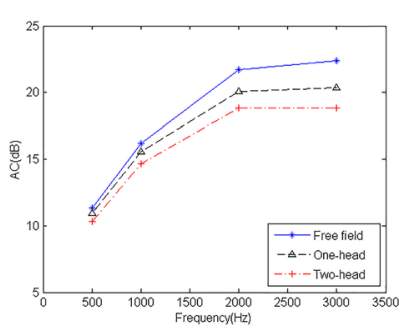

(c)

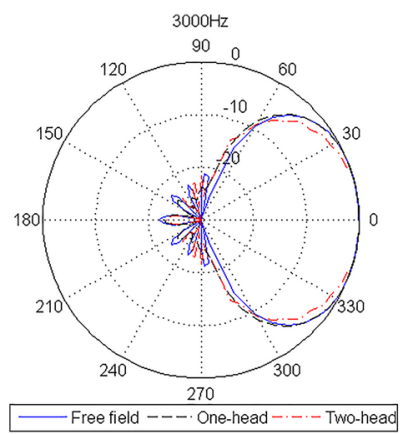

(b)

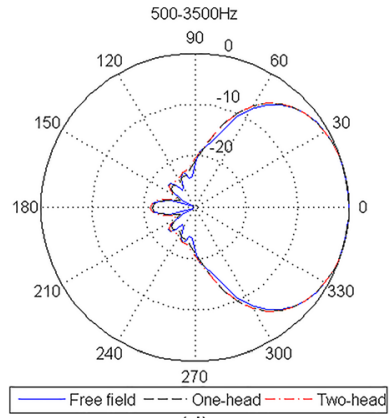

(d)
FIG. 3. (Color online) Simulation results with (a) single-frequency directivity at $500 \mathrm{~Hz}$, (b) single-frequency directivity at $3000 \mathrm{~Hz}$, (c) AC (acoustic contrast) varied with frequency, and (d) broadband directivity. The free field performance is shown by the solid line, one-head scattering performance is shown by the dash line, and two-head scattering performance is shown by the dash-dot line. 


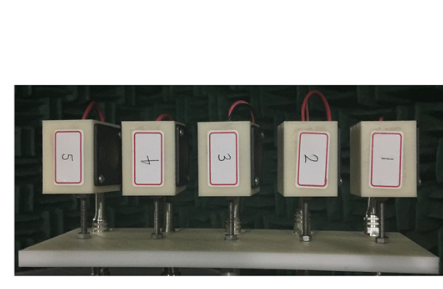

(a)

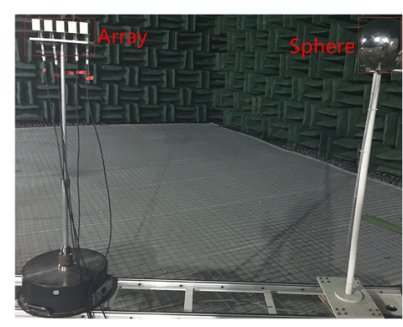

(c)

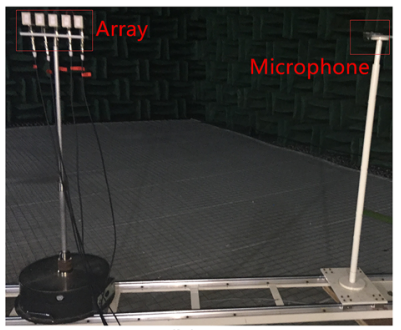

(b)

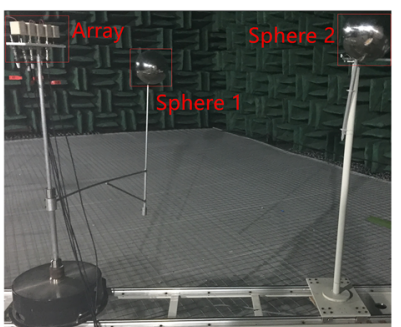

(d)

FIG. 4. (Color online) (a) The five-loudspeaker compact array, (b) the free field experiment, (c) one-head scattering experiment, (d) two-head scattering experiment.

$$
\mathbf{q}_{\text {adjusted }}(f)=\frac{\mathbf{q}_{\mathrm{op}}(f)}{\left|p_{\mathrm{b}, \text { center }}(f)\right|},
$$

where $\mathbf{q}_{\text {op }}(f)$ is the optimal source strength at the frequency $f$, and $p_{\mathrm{b} \text {,center }}(f)$ is the sound pressure at the center sampling point in the bright zone. Finally, the time domain FIR filter can be obtained using the inverse Fourier transform, and each filter is delayed 80 points to guarantee the causality of the system.

The simulation results are shown in Fig. 3, where it shows that the AC increases with the frequency under both the free field and the scattering conditions. Compared to the free field result, the scattering has barely any influence at $500 \mathrm{~Hz}$ shown in Fig. 3(a) while the influence at $3000 \mathrm{~Hz}$ can be observed in Fig. 3(b). 2.0 and $3.5 \mathrm{~dB}$ reduction of the $\mathrm{AC}$ at $3000 \mathrm{~Hz}$ can be found in Fig. 3(c) for one-head and twohead scattering, respectively. The influence of the head scattering does exist but is limited even at frequency as high as $3000 \mathrm{~Hz}$. The broadband performance shown in Fig. 3(d) further demonstrates the robustness of the system to the head scattering effects. Note that similar results are obtained when the radius of the evaluation zone is $0.5 \mathrm{~m}$. Therefore, this compact system is expected to be a good personal audio system for speech signal reproduction.

\section{EXPERIMENTS}

A five-loudspeaker compact array experimental system as described in Sec. II was designed and the experiments were carried out in an anechoic chamber. Figures 4(a)-4(d) show the compact array system together with three experimental configurations. Before the experiment, the five loudspeakers were calibrated using the measured transfer function at $1.0 \mathrm{~m}$. The compact array was placed on a turntable, the directivity of the array was measured and the AC was calculated.

The experimental results are shown in Figs. 5(a)-5(d), where the performance variation caused by the sphere scattering is found to be larger than that in the simulations.

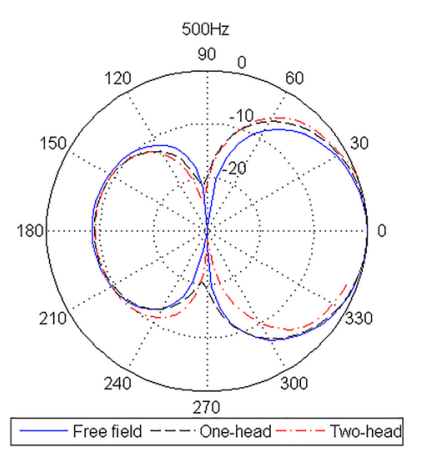

(a)

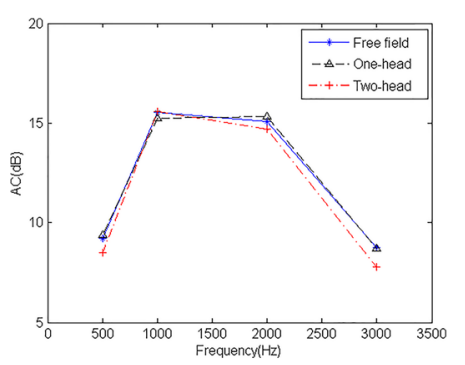

(c)

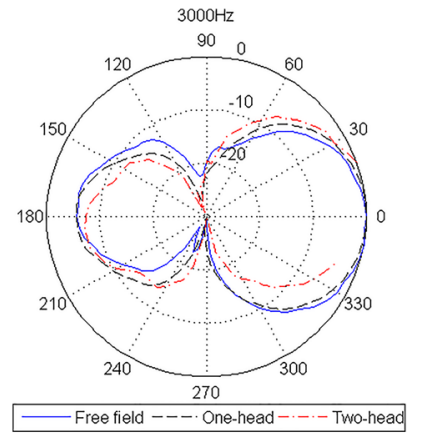

(b)

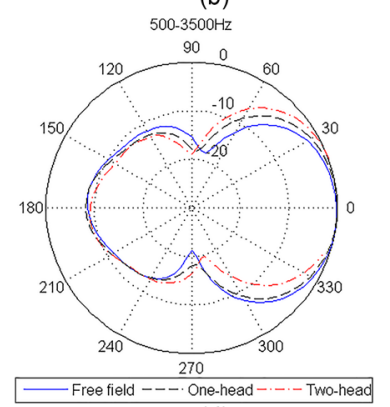

(d)
FIG. 5. (Color online) Experimental results with (a) single-frequency directivity at $500 \mathrm{~Hz}$, (b) single-frequency directivity at $3000 \mathrm{~Hz}$, (c) AC (acoustic contrast) varied with frequency, and (d) broadband directivity. The free field performance is shown by the solid line, one-head scattering performance is shown by the dash line, and two-head scattering performance is shown by the dash-dot line.

However, careful investigation depicts that the variation is mostly caused by the non-symmetric directivity as a result of the bias of the sphere center in the experiments. Furthermore, the ACs shown in Fig. 5(c) are very close to each other with only 0.1 and $1.0 \mathrm{~dB}$ reduction of the $\mathrm{AC}$ at $3000 \mathrm{~Hz}$ for one-head and two-head scattering scenarios, respectively. Therefore, the robustness of the system to the head scattering effects is verified.

Compared to the simulation results, the measured array performance deteriorates significantly at high frequencies, especially at about $180^{\circ}$. This is mainly caused by the variation of the acoustic center of each loudspeaker and the considerable scattering of the loudspeakers themselves especially at high frequencies. On the other hand, the AC values of the experiment are very similar to that of the simulation at some frequencies, such as $500 \mathrm{~Hz}$, but the directivity shows significant difference. This is because the sound pressure level in the experiment is higher in the range of $100^{\circ}-260^{\circ}$, but lower at other degrees, which can be found by carefully investigating Figs. 3(a) and 5(a). However, the acoustic focusing of the system is still obvious and the system is quite robust to the head scattering effects.

\section{CONCLUSIONS}

Based on the regularized acoustic contrast control method, this paper uses a five-loudspeaker compact array system with bright zone at the endfire direction to investigate the robustness of the system to the scattering effects caused by the listeners' heads. Two scenarios are investigated, and the first one has a listener moving around the interested 
zone, while the second one has one listener standing in the bright zone and another one moving along the circle. Both simulation and experiment results demonstrate that the scattering has limited influence in both the two scenarios on the whole directivity performance of the endfire system.

\section{ACKNOWLEDGMENTS}

This work was supported by National Natural Science Foundation of China Grant Nos. 11374156 and 11474163.

${ }^{1}$ T. Betlehem, W. Zhang, M. A. Poletti, and T. D. Abhayapala, "Personal sound zones: Delivering interface-free audio to multiple listeners," IEEE Signal Process. Mag. 32(2), 81-91 (2015).

${ }^{2}$ P. Coleman, P. J. B. Jackson, M. Olik, M. Olsen, M. Møller, and J. A. Pedersen, "Acoustic contrast, planarity and robustness of sound zone methods using a circular loudspeaker array," J. Acoust. Soc. Am. 135(4), 1929-1940 (2014).

${ }^{3}$ J. W. Choi and Y. H. Kim, "Generation of an acoustically bright zone with an illuminated region using multiple sources," J. Acoust. Soc. Am. 111(4), 1695-1700 (2002).

${ }^{4}$ O. Keikeby and P. A. Nelson, "Reproduction of plane wave sound fields," J. Acoust. Soc. Am. 94(5), 2992-3000 (1993).

${ }^{5}$ M. R. Bai and Y. H. Hsieh, "Point focusing using loudspeaker arrays form the perspective of optimal beamforming," J. Acoust. Soc. Am. 137(6), 3393-3410 (2015).

${ }^{6}$ J. H. Chang and F. Jacobsen, "Sound field control with a circular doublelayer array of loudspeakers,” J. Acoust. Soc. Am. 131(6), 4518-4525 (2012).
${ }^{7}$ M. Shin, F. M. Fazi, P. A. Nelson, and F. C. Hirono, "Controlled sound field with a dual layer loudspeaker array," J. Sound Vib. 333(16), 3794-3817 (2014).

${ }^{8}$ S. J. Elliott, J. Cheer, J. W. Choi, and Y. H. Kim, "Robustness and regularization of personal audio systems," IEEE Trans. Audio, Speech, Lang. Process. 20(7), 2123-2133 (2012).

${ }^{9}$ J. Cheer, S. J. Elliott, Y. H. Kim, and J. W. Choi, "Practical implementation of personal audio in a mobile device," J. Audio Eng. Soc. 61(5), 290-300 (2013)

${ }^{10}$ M. F. S. Gálvez, S. J. Elliott, and J. Cheer, "A superdirective array of phase shift sources,” J. Acoust. Soc. Am. 132(2), 746-756 (2012).

${ }^{11}$ P. Coleman, M. Moller, M. Olsen, M. Olik, P. J. B. Jackson, and J. A. Pedersen, "Performance of optimized sound field control techniques in simulated and real acoustic environments," J. Acoust. Soc. Am. 131(4), 3465-3465 (2012)

${ }^{12}$ J. Y. Park, J. W. Choi, and Y. H. Kim, “Acoustic contrast sensitivity to transfer function errors in the design of a personal audio system," J. Acoust. Soc. Am. 134(1), EL112-EL118 (2013).

${ }^{13}$ M. F. S. Gálvez, S. J. Elliott, and J. Cheer, "The effect of reverberation on personal audio devices," J. Acoust. Soc. Am. 135(5), 2654-2663 (2014).

${ }^{14}$ J. H. Chang, J. Y. Park, and Y. H. Kim, "Scattering effect on the sound focused personal audio system," J. Acoust. Soc. Am. 125(5), 3060-3066 (2009).

${ }^{15}$ J. H. Chang and F. Jacobsen, "Experimental validation of sound field control with a circular double-layer array of loudspeakers," J. Acoust. Soc. Am. 133(4), 2046-2054 (2013).

${ }^{16} \mathrm{~B}$. Masiero and X. Qiu, "Two listeners crosstalk cancellation system modelled by four point sources and two rigid spheres," Acta Acust. Acust. 95(2), 379-385 (2009).

${ }^{17}$ J. W. Choi and Y. H. Kim, "Active control for the enhancement of sound field," in Proceedings Active' 04, Williamsburg, VA (2004). 